\title{
Editorial
}

\section{Contributions of Sasang Constitutional Medicine}

\author{
Edwin L. Cooper \\ Department of Neurobiology, David Geffen School of Medicine at UCLA, University of California, Los Angeles, \\ CA 90095-1763, USA
}

Sasang Constitutional Medicine (SCM), also referred to as 'integrative medicine', constitutes a unique contribution to the growing field of complementary and alternative medicine. Like all other ancient cultures-including those from India, (Ayurveda) China (TCM), Japan (Kampo), Korea (TKM) and the Mediterranean (TAIM) - there is an emerging fascination with, and indeed use of, these approaches as adjuncts to Western medicine and often as alternatives. This special supplement to $e C A M$ represents a milestone, in that it is the first publication in this area of alternative medical practices to be published. The papers were first written and subjected to internal peer review by scientists at the Korea Institute of Oriental Medicine in Daejeon, Korea (KIOM). The very best papers were then chosen and submitted by the usual route to $e C A M$. They were then subjected to further intense international peer review and underwent revisions based upon the referees' comments. What is presented here consists of the very best papers that survived the internal KIOM review process as well as the scrutiny of the anonymous referees and members of the editorial board of $e C A M$.

In keeping with the tradition of $e C A M$, for each publication there was a strong effort to present information in clear English, often requiring the certification of a licensed expert familiar with transparent and scientific English, followed by the necessity to impose certain stylistic suggestions to improve the appearance and general readability of papers. In all instances, authors were urged to consider supplementing the text with appropriate hypothetical drawings that best explain newly presented results and propose future approaches to validate certain

For reprints and all correspondence: Edwin L. Cooper, Department of Neurobiology, David Geffen School of Medicine at UCLA, University of California, Los Angeles, CA 90095-1763, USA. claims. This not only helps authors, but it also provides clarity for readers and useful information important in teaching and for presentation at various meetings. The entire supplement is organized and presented as Reviews and Original Articles of two types: Basic Science and Clinical Analyses, the usual style of $e C A M$.

The introductory paper contributed by Prof. Denis Noble sets the stage for the entire supplement. In his words, "[a]ttitudes towards oriental medicine are changing for two major reasons. First, many patients, even in the West, are choosing to use its practitioners and methods. Second, the rise of oriental and Western traditions; much work is required to facilitate dialogue and synthesis. There should be time devoted to clarifying meanings of terms and the framework of theory and practice within which oriental methods operate. Moreover it is necessary for Systems Biology just emerging itself to mature as a discipline, particularly at the higher levels of biological organization since it is at these levels that oriental medicine derives its ideas and practice. Higher level Systems Biology could then be a basis for interpreting the Korean version of oriental medicine: Sasang constitutional medicine since it seeks patient specific analysis and treatment, and the mathematical methods of systems biology could be used to analyze the central concept of balance in Sasang.'

Although Noble mentions Systems Biology and early citations of Claude Bernard, I would like to mention one of the first recent Western citations involving the term and its utility to biomedicine. According to Hood et al. (1), the new 'Systems approaches to disease are grounded in the idea that disease-perturbed protein and gene regulatory networks differ from their normal counterparts; we have been pursuing the possibility that these differences may be reflected by multiparameter measurements of the blood. Such concepts are transforming the current diagnostic and therapeutic approaches to 
medicine and, together with new technologies, will enable a predictive and preventive medicine that will lead to personalized medicine.'

Actually, several publications in the form of lectures take precedent over KIOM and may bear some similarities. In the second year of $e C A M$, I agreed to assist Jose Olalde Rangel to publish his treatise. According to Olalde Rangel (2-5), the systemic theory postulates that health $(\mathrm{H})$ is directly proportional to the integrity of a living system's energy (E), biointelligence (I) and organization (O). Systemic theory also establishes a common denominator to all sickness and ascertains the cause of all disease to be an entropy increase: 'disorder augmenting within the biologically open system, stemming from energy-informational and organizational impacts, either of external or internal nature. Therapeutics should then include a negentropy supply to enhance the system's energy-work capacity (E), its informational potential (I) intelligence, and finally structure and functional organization (O). Systemic Medicine's (SM) treatment strategy is based on identifying and prescribing superior herbs-tonic or adaptogenic- or any nutraceuticals or medicine with potential to strengthen E, I, O by providing energy, informational and organizational aid to the overall network of intelligent cells and cell systems that constitute the body. The main premise proposes that when all three factors are brought back to ideal levels patient's conditions begin recovery to normal health.'

Now to the specifics of this KIOM Supplement. First, there are six Reviews on general constitutional medicine. In 'Sasang Constitutional Medicine as a Holistic Tailored Medicine', authors propose that two main axes in the physiopathology of SCM are equivalent to the process of internal-external exchange and catabolism/anabolism in modern physiology. They continue to state that the constitutional approach of SCM shares the same vision with tailored medicine. 'Psychological Profile of Sasang Typology: A Systematic Review' shows that the results of the review indicate that two super-factors, Extraversion and Neuroticism, serve as the foundation of delineating personality constructs, such that the So-Yang type scores high on the Extraversion dimension and low on the Neuroticism dimension, while the So-Eum type scores low on the Extraversion dimension and high on the Neuroticism dimension.

The authors of 'Perspective of the Human Body in Sasang Constitutional Medicine' explain the basic theory of SCM in detail. In 'Current Researches on the Methods of Diagnosing Sasang Constitution: An Overview', the authors searched the Journal of Sasang Constitutional Medicine, other Korean domestic journal databases and PubMed for research regarding modernized constitution diagnosis methods so as to provide the understanding of current research state and outlook for future research. 'Genetic Approach to Elucidation of Sasang Constitutional Medicine' describes the attributes of the constitution concept of SCM that can be interpreted in the language of genetics and current approaches to identify the genetic factors that make up the constitution. 'Randomized Clinical Trials of Constitutional Acupuncture: A Systematic Review' is to compile and critically evaluate the evidence from randomized clinical trials for the effectiveness of acupuncture using constitutional medicine compared with standard acupuncture.

We present three publications in the section of Basic Science. 'Feature Selection from a Facial Image for Distinction of Sasang Constitution' is to establish methods for finding statistically significant features in a facial image with respect to distinguishing constitution and to show the meaning of those features. In 'Association between Genetic Polymorphism of Multidrug Resistance 1 Gene and Sasang Constitutions', authors show significant differences in allele distribution between So-yangin type and Tae-eumin type. So-yang type and So-eum type showed significant genetic differences between constitutions. 'Effects of Taeyeumjoweetang on Body Weight and Obesity-related Genes in Mice' explains that an herb remedy (TYJWT) has obesity-suppressing effects similar to those previously reported using highfat diets.

For Clinical Analyses, there are also three representative articles. 'Analysis of Skin Humidity Variation between Sasang Types' examines the relationship between variations in skin humidity induced by perspiration across Sasang types. 'Quantitative Sasang Constitution Diagnosis Method between Tae-eumin and Soeumin Types through Elasticity Measurements of the Skin of the Human Hand' compares the skin elasticity between Soeumin and Tae-eumin subjects. 'Sasang Constitution as a Risk Factor for Diabetes Mellitus: A Cross-sectional Study' shows that constitution has a significant and independent association with diabetes, which suggests that constitution is an independent risk factor for diabetes that should be considered when attempting to detect and prevent the disease.

In summary, clearly SCM represents a potentially fruitful modern approach to an ancient practice that will probably attract more and more attention. This reciprocal stimulation will derive from workers in areas other than the precisely defined complementary and alternative medicine, i.e. Western medicine, in other areas with appropriate congruence and overlap. For example, there are clear psychological overtones that could be embraced by practicing psychologists; and, for the more clinically oriented, the medically trained psychiatrist will surely find numerous areas of similarity that may offer a new approach to diagnosis. Moreover, sociologists could embrace ideas that might even overlap into diagnosis of potential law breakers. Thus, the application seems most promising. 


\section{References}

1. Hood L, Heath JR, Phelps ME, Lin B. Systems biology and new technologies enable predictive and preventative medicine. Science 2004;306:640-3.

2. Olalde Rangel JA. The systemic theory of living systems and relevance to CAM. Part I: the theory. Evid Based Complement Alternat Med 2005;2:13-18.

3. Olalde Rangel JA. The systemic theory of living systems and relevance to CAM. Part II: the theory. Evid Based Complement Alternat Med 2005;2:129-37.
4. Olalde Rangel JA. The systemic theory of living systems and relevance to CAM. Part III: the theory. Evid Based Complement Alternat Med 2005;2:267-75.

5. Olalde Rangel JA, Magarici M, Amendola F, del Castillo O. The systemic theory of living systems. Part IV: systemic medicine-the praxis. Evid Based Complement Alternat Med 2005;2:429-39. 


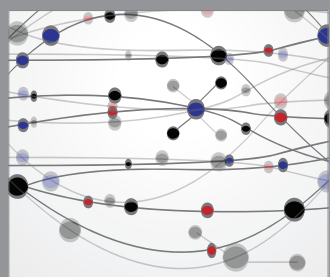

The Scientific World Journal
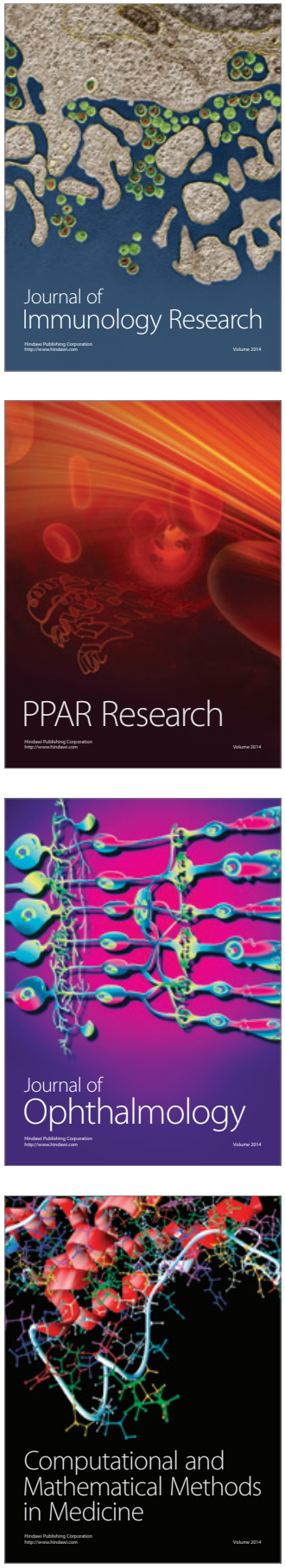

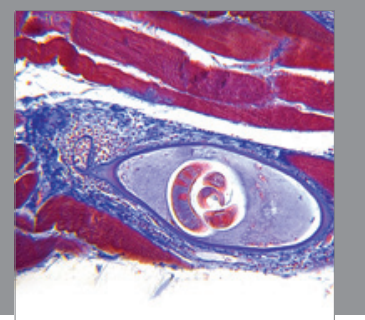

Gastroenterology

Research and Practice
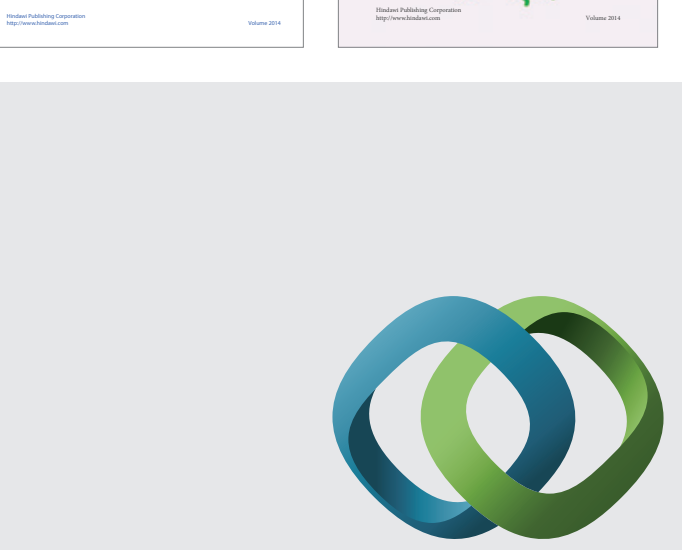

\section{Hindawi}

Submit your manuscripts at

http://www.hindawi.com
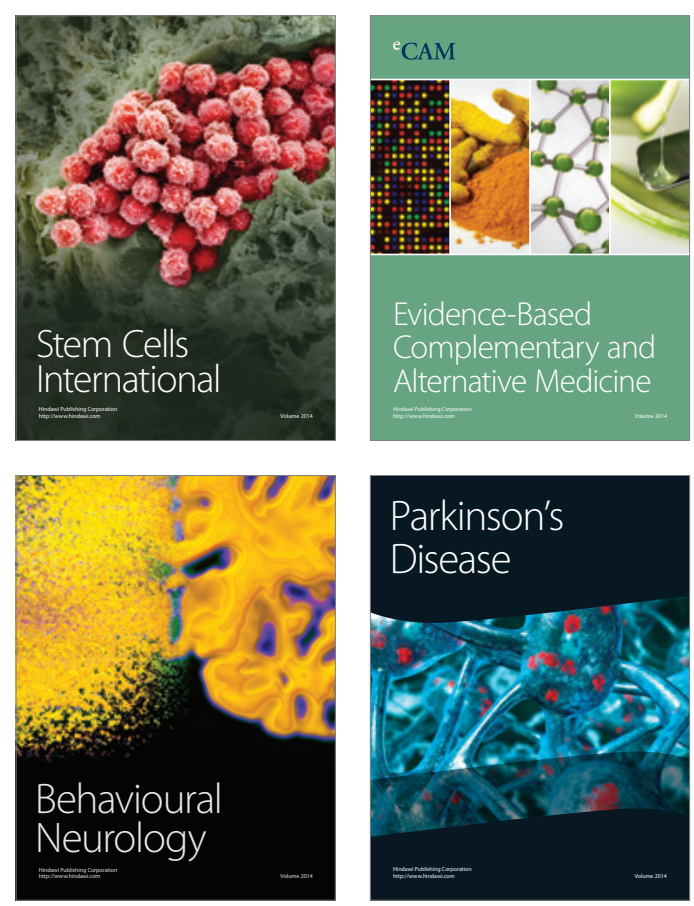

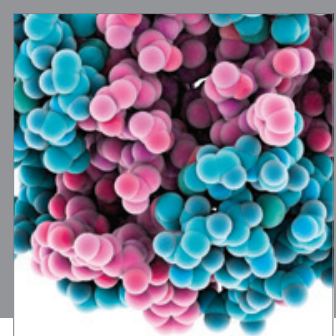

Journal of
Diabetes Research

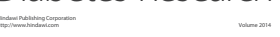

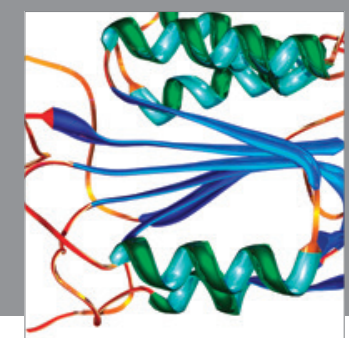

Disease Markers
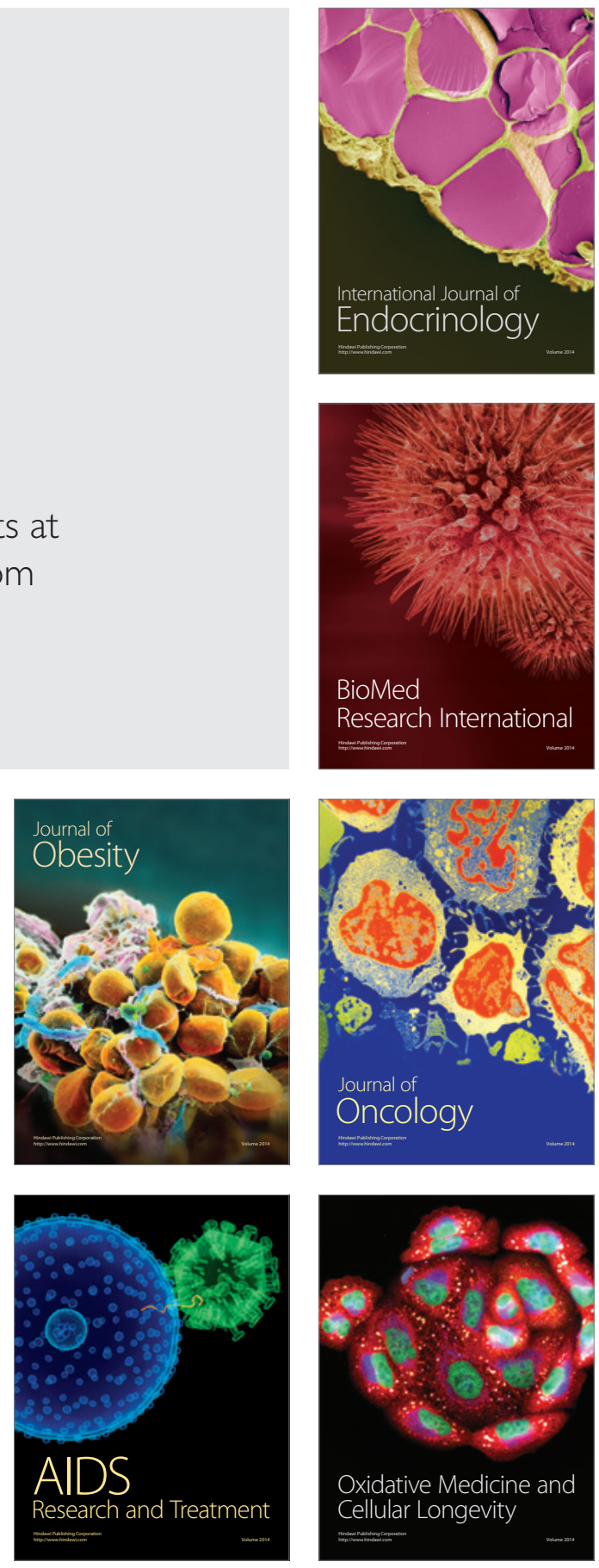